\title{
Return of EEG activity after electrocerebral silence: two case reports
}

\author{
JOSEPH B. GREEN AND ANDRE LAUBER \\ From the Department of Neurology, Indiana University School of Medicine, \\ 1100 West Michigan Street, Indianapolis, Indiana 46202, U.S.A.
}

SUMmaRY Two cases, both children, are described, in which there was a return of the electroencephalogram after a period of electrocerebral silence. One child survived for six weeks. Electroencephalographic technique and instrumentation adequately excluded non-cerebral potentials as a source of confusion in the second case. Hypothermia and drug overdosage, known to reversibly depress the electroencephalogram, were not present in either case. The return of EEG activity was associated with improvement in neurological status. It is concluded that the EEG should always be repeated after electrocerebral silence before the determination of cerebral death, and that the applicability of adult criteria of brain death to children is questionable.

The recent survey of the American EEG Society's Ad Hoc Committee on EEG Criteria for the Determination of Cerebral Death (Silverman, Masland, Saunders, and Schwab, 1970) revealed no acceptable example of recovery of cerebral function after a truly isoelectric electroencephalogram (EEG) except for three cases involving massive drug overdosage (Bird and Plum, 1968). No consensus was reached on the time electrocerebral silence should last before irreversibility can be concluded, so the Committee recommended that the EEG be repeated within 24 hours. They stated that this 'conservative' recommendation was necessary since the maximum revival time after an anoxic insult in the human being is not known. Becker, Robert, Nelson, and Stern (1970), question the requirement that a period of 24 hours must always elapse, and note that in order to provide well-perfused organs for donation it may be urgent to declare certain patients cerebrally dead short of this time span.

The necessity for repeating the EEG after an initial isoelectric record has been recently demonstrated in this laboratory. In two cases, both children, there was apparent return of cerebral electrical activity 24 hours after 'electrocerebral silence'. Follow-up EEGs showed persistence of activity in one of these children who survived for six weeks.

\section{CASE 1}

A 5 year old boy had been well until three months before admission, when he became easily fatigued and anorexic. Jaundice and dark urine followed and resulted in hospitalization locally. His liver was enlarged and tender, and tests of liver function revealed a rise in bilirubin to $10.3 \mathrm{mg} / 100 \mathrm{ml}$., an alkaline phosphatase of 7.8 units, total protein of $8.4 \mathrm{gm} / 100 \mathrm{ml}$., with 2.8 of that albumin, and an SGOT of 10,000 units. After transfer to the Indiana University Medical Center he failed to improve and became irritable and less communicative. He became confused and agitated. Neurological examination revealed rigidity of the lower extremities, bilateral ankle clonus, and Babinski signs. A beating tremor (asterixis) was observed. Despite several exchange transfusions the child's condition continued to deteriorate and on the 12th day after transfer he was discovered in coma, with dilated and fixed pupils. One day later he had an arrest of respiration and was ventilated artificially with monitoring of blood gases. He failed to improve but became flaccid in all extremities, with hypoactive muscle stretch reflexes. He failed to react to any stimulation. An EEG at this time showed no electrical activity 'attributable to the cerebrum'. The record was taken for 30 minutes (running time), and with bipolar and monopolar electrode placement. The gain was at the highest setting (Fig. 1).

During the 24 hours after the first EEG there were some clinical signs which suggested improvement. The patient withdrew from painful stimuli to the feet, legs, and abdomen. There were spontaneous movements of the extremities. The muscle stretch 


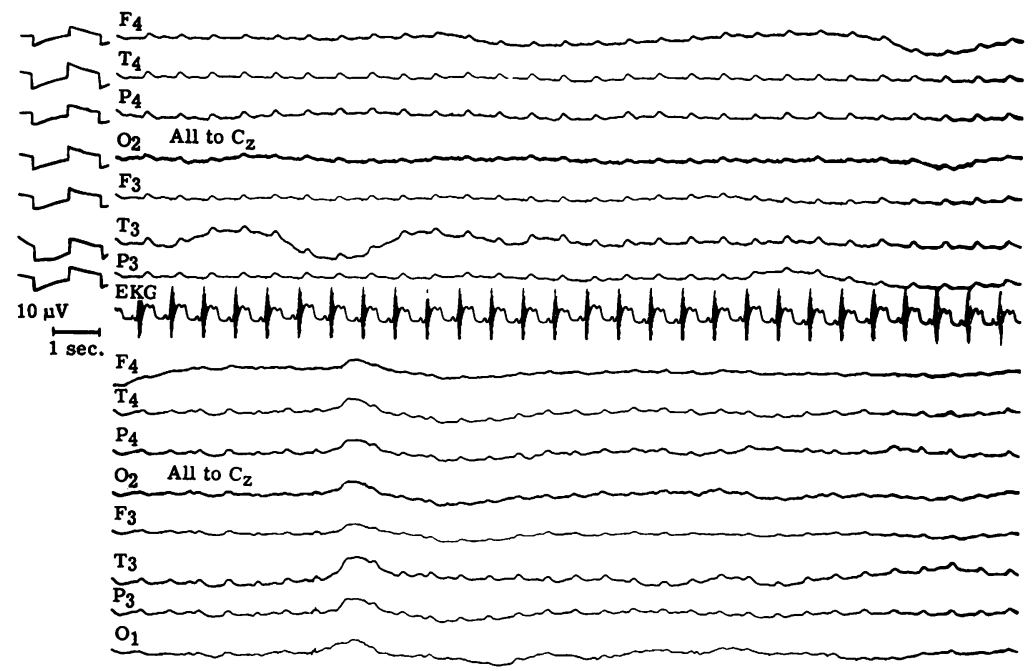

FIG. 1. Case 1. EEG recorded at highest gain setting $(S=2)$. Low Linear Frequency Filter set at 1 . (Grass Model VI electroencephalograph.)

reflexes became active. Repeat EEG exactly 24 hours after the initial recording showed fast activity of 2 to $4 \mu \mathrm{V}$. This was present intermittently and on either side; at times some bilateral activity was recorded (Fig. 2).

The patient's urinary output ceased and he became oedematous. He expired seven hours after the second EEG. At necropsy the cerebrum was softened and oedematous and was reported to show microscopic changes 'typical of respirator brain'.

\section{CASE 2}

A 6 week old male infant was admitted because of seizures and apnoea. One day before admission his mother found him with eyes rolled upward, arms twitching, and without respiration for an unknown period. He was cyanotic but recovered as she watched. He lapsed into apnoea two more times? before reaching the hospital. On admission he was or 'moribund', stiff, and dehydrated. Electrolyte values - and other blood chemistry determinations wege $\vec{c}$ compatible with dehydration. Cerebrospinal flumit studies were unremarkable. The child was give phenytoin (Dilantin) and phenobarbitone, each in $a-$ dosage of $5 \mathrm{mg} / \mathrm{kg}$ and $2 \mathrm{mg}$ diazepam (Valium) $\mathbb{D}$ intravenously. He improved, and examination the second day showed a suck reflex; he cried, and hog good colour. The anterior fontanelle was small an soft. Pupils reacted to light and extraocular move ments were full. There was spasticity bilaterally with ? the muscle stretch reflexes more active on the right and bilateral Babinski signs. He withdrew extremities on painful stimulation. Heart rate was 150 and

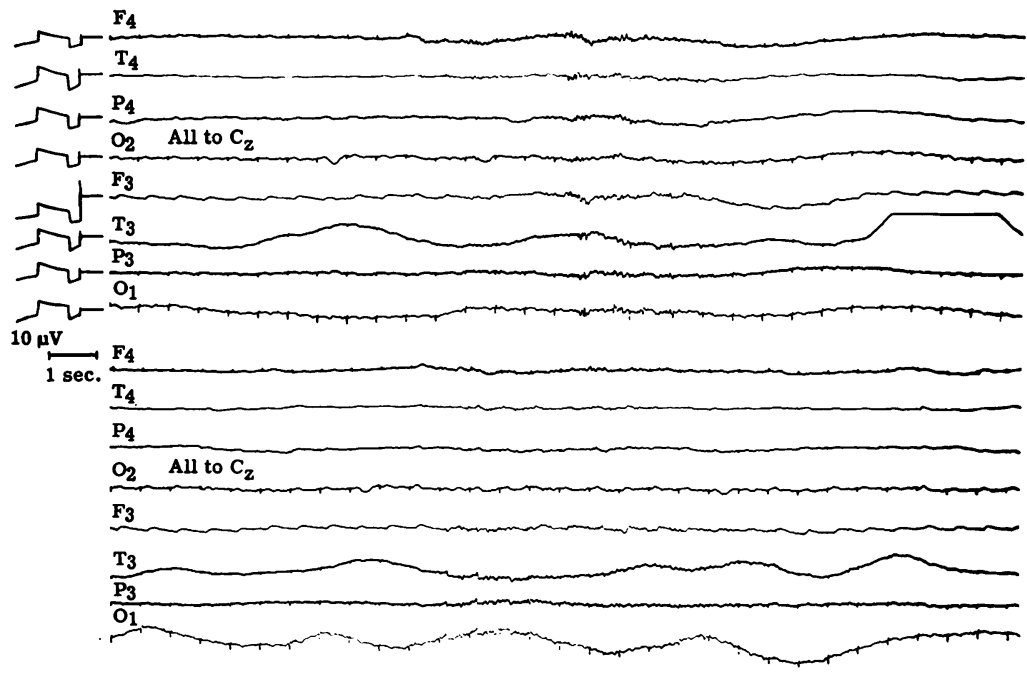

FIG. 2. Case 1. EEG recorded 24 hours after electrocerebral silence (Fig. 1). Note fast rhythm of $18 \mathrm{~Hz} . S=2, L L F=1$. 
respirations were 35 per minute, temperature was $35 \cdot 2^{\circ} \mathrm{C}\left(95 \cdot 4^{\circ} \mathrm{F}\right)$, and weight was $5 \mathrm{~kg}(11 \mathrm{lb} .6 \mathrm{oz})$.

The patient had been born at 38 weeks' gestation after precipitous labour following an automobile accident. The cord was 'wrapped around the neck three times'. Birth weight was $3 \mathrm{~kg}(6 \mathrm{lb} .11 \mathrm{oz})$. The child had a poor sucking reflex and Moro reflex for the first 12 hours of life. He had apparently recovered completely from this post-natal depressed state and had been well until the day preceding admission.

Several more seizures occurred during the second day of hospitalization and consisted of blinking of the eyes and jerking of the right hand. It was necessary to intubate him and assist respiration with a Bourns ventilator. Radiographs of the chest showed a right upper lobar infiltration. On the third day 75 to $80 \%$ of the right upper lobe was collapsed and there was an infiltration on the left side. Electrolytes, including calcium and magnesium, were within normal limits and blood gas partial pressures and $\mathrm{pH}$ were maintained within satisfactory range. Serum phenytoin level was only $1 \cdot 1 \mu \mathrm{g} / \mathrm{ml}$. and phenobarbitone was $4.0 \mu \mathrm{g} / \mathrm{ml}$.

He had received only a single $25 \mathrm{mg}$ dose of Dilantin intramuscularly after the first day of hospitalization and no phenobarbitone or Valium.

On the fourth day there was a decreased output of

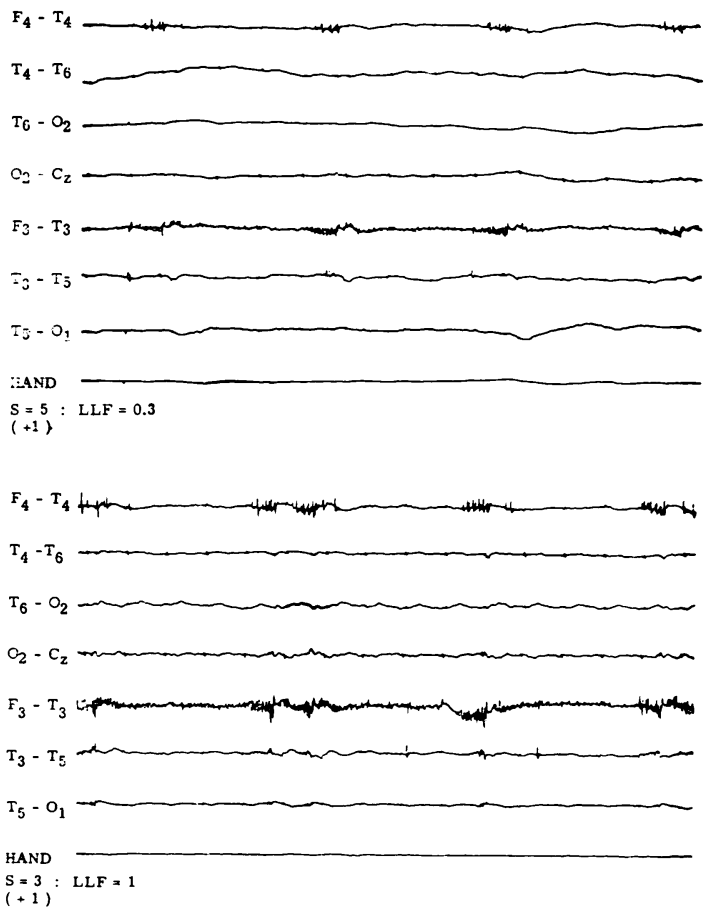

FIG. 3. Case 2. First EEG recorded with bipolar montage. No cerebral electrical activity. Hand: two electrodes on dorsum of hand to control for 'noise'.

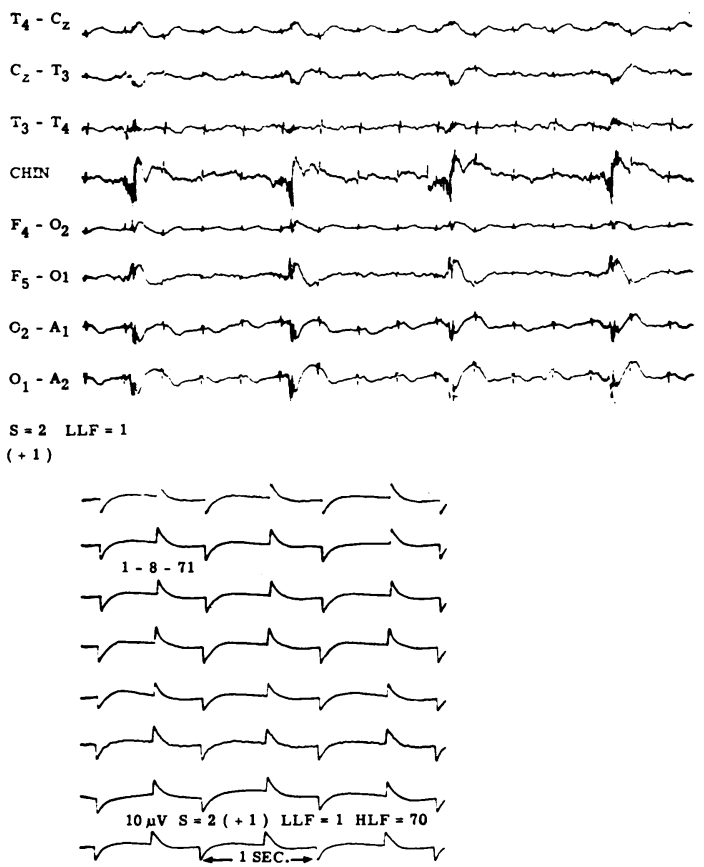

FIG. 4. Case 2. First EEG recorded with monopolar and bipolar montage. 'Chin' represents pair of electrodes on chin to control for "noise' level and also to record a reflex twitch produced by the respirator. Sensitivity set at maximum. No cerebral electrical activity is present. Calibration is for this figure and Fig. 3.

urine. Pupils were fixed and dilated. The extremities were flaccid with absent stretch reflexes. There was no response to painful stimuli and no spontaneous respiration. An EEG performed in accordance with recommended techniques showed no cerebral electrical activity at the highest gain setting (Figs 3 and 4). On the following day a repeat EEG showed 12 to $18 \mathrm{~Hz}$ rhythmical activity, sharp waves, and slow activity (Figs 5 and 6). That same day the child moved his extremities spontaneously, again withdrew from painful stimuli, and regained pupillary reaction to light. During the following two days muscle stretch reflexes also returned and the child made sucking and chewing movements. He opened his eyes on occasion. Two more EEGs, recorded 72 and 96 hours after the initial isoelectric record, showed a variety of frequencies present. An EEG performed 20 days later recorded spikes and an apparent response to photic stimulation (Fig. 7).

There was little change in his clinical condition until the 20th day of hospitalization when the child regained spontaneous respiration. $\mathrm{He}$ was able to move his eyes conjugately and suck for the first time since admission. He continued in this state for three weeks, then died suddenly and unexpectedly. 


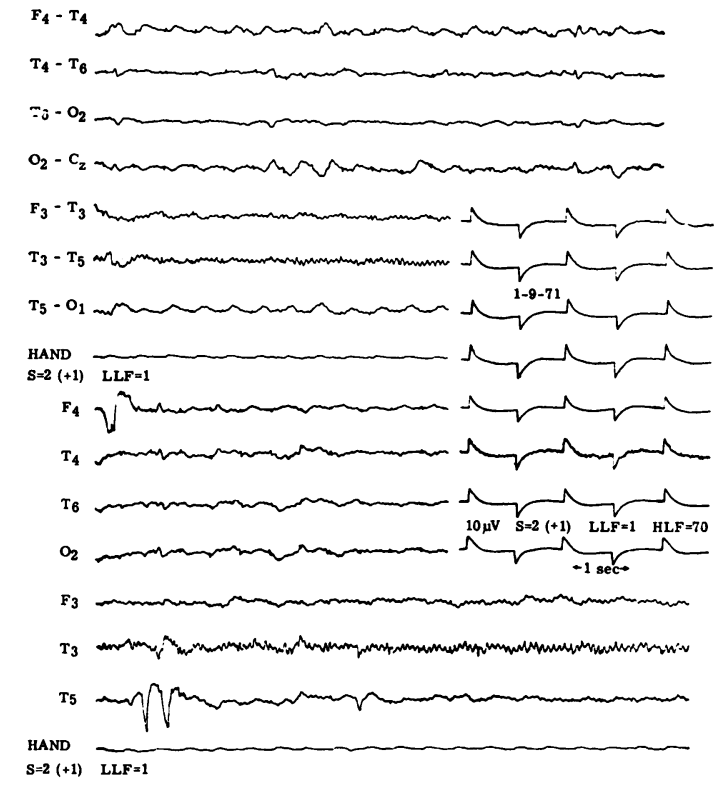

FIG. 5. Case 2. Second EEG recorded 24 hours after first showed fast activity at $18 \mathrm{~Hz}$ with no 'noise' present in hand electrodes. Slow waves also are visible.

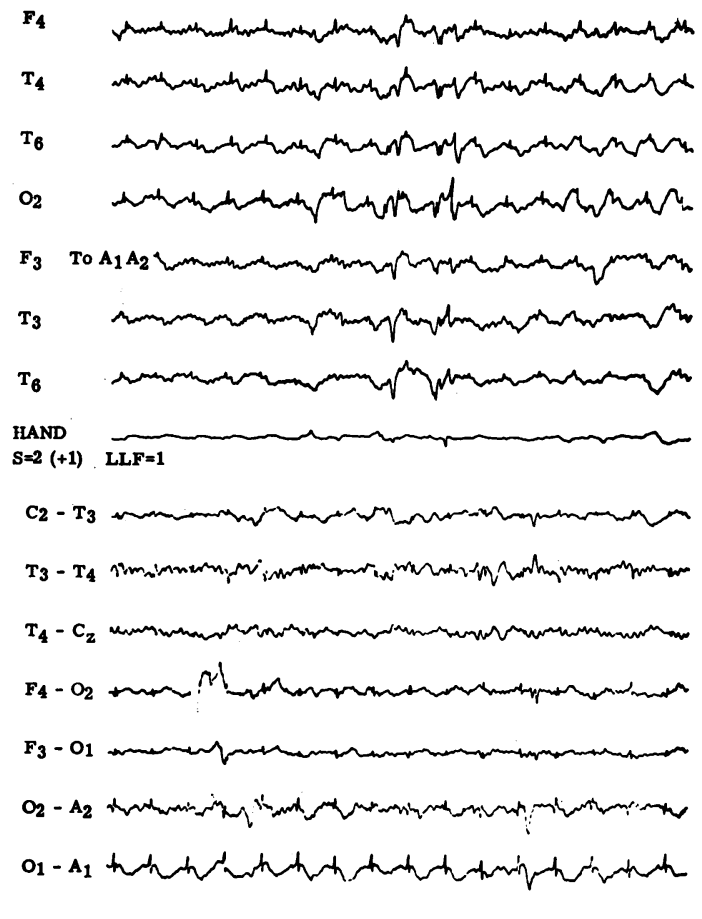

HAND

$S=2(+1) \quad L L F=1$

FIG. 6. Case 2. Second EEG showing spikes, slow waves, and fast activity with silent hand electrodes. Calibration for this Figure also for Fig. 3.

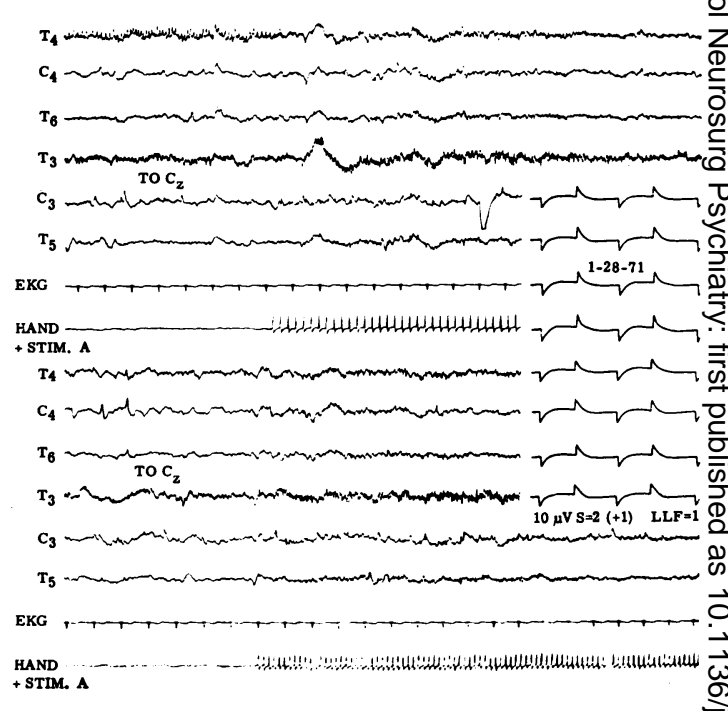

FIG. 7. Photic stimulation influenced on-going ac tivity. Note silence in hand electrodes (stimulus artefact $=$ Stim. $A$ is also introduced in this channel) Note spikes in $T_{4}$ and $C_{4}$ electrode locations.

Necropsy revealed changes of pneumonitis andS congestion. The brain showed multiple porenceplar lies and ulegyria. The cortex was thinned in ares overlying cavitated white matter. There was cerebellar sclerosis and sclerosis of the basis pontis agido medullary pyramids. The neuropathologist thoug $k$ the changes were consistent with a perinatal aetiolog, $\overrightarrow{0}$ rather than the result of the more recent apnoea and the supportive measures (respirator).

\section{DISCUSSION}

The evidence for the return of EEG activity in case 1 is inconclusive. Unfortunately, an extra- $\overrightarrow{\vec{\theta}}$ cranial pair of electrodes was not employed-as, $\exists$ for example, on the dorsum of the right handwhich would have excluded machine noise, radiated interference, and vibration as a cause for the beta-like waves in the second (repeat)? EEG. The patient did improve between EEGs before his death from renal failure. He began to withdraw from painful stimuli, moved spontaneously, and had a return of muscle tone and stretch reflexes.

In case 2 there would seem clearly to be activity present in three electroencephalograms after an isoelectric tracing. Bipolar montages of with adequately long interelectrode distances 0 were employed, as well as ear and vertex refer- $-\tilde{\omega}$ ences. Electrode resistances were under 3,000 $\Omega$, but over 100 , and touching electrodes producedo 
an artefact. A portion of each record was run at the highest gain setting of the instrument and included use of a time constant of 0.4 seconds.

Neither scalp nor extracranial leads in case 2 showed any activity spontaneously or on stimulation (pain, sound, or light) during the first EEG. The second record 24 hours later showed activity confined to the scalp leads reminiscent of the fast activity present in case 1 . Moreover, two subsequent EEGs showed even more activity with sharp waves and theta rhythms. There had been no hypothermia in case 2 at the time of the initial record (which may reversibly depress cerebral activity) and drugs were evidently not a factor, since levels of Dilantin and phenobarbitone were not high. Anaesthetic doses of central nervous system depressants were clearly not implicated in this recovery from electrocerebral silence.

The return of activity to the EEG correlated with clinical improvement in each case, with return also of muscle tone and reflexes. Actually in case 2 the improvement in the child's neurological status was preceded by recovery of the EEG. This coincidence of EEG and clinical improvement provides indirect evidence for the validity of the EEG findings.

Technical considerations in EEG recordings have been emphasized by Silverman, Saunders, Schwab, and Masland (1969), and Silverman et al. (1970) and rightly cast doubt upon several reports of recovery after isoelectric EEGs. Lindgren, Petersén, and Zvetnow (1968) have reported such a case but in their illustrations they do not indicate they used a sensitivity setting which was adequate to exclude activity in the initial record. This objection is not applicable in our two cases, although an extracranial monitor was not used in case 1 .

Surprisingly, slow waves were not the first activity to return in our cases, but rather frequencies of 10 to $18 \mathrm{~Hz}$. Bursts at this frequency are present in the immature brain and occasionally tonic seizures are accompanied by similar activity. Rhythm of this type may be recorded in the malformed brain of holoprosencephaly, but voltage is much higher (DeMyer and White, 1964). It is conceivable that the fast activity in case 2 represented seizure activity since the child had a history of attacks.

The several reports concerning the criteria for the determination of brain death make no distinction between adults and children (Harvard Medical School, 1968), despite known differences in their response to hypoxic insults (Windle, 1964). It may be the lessened susceptibility of the child's brain to anoxia which permitted an apparently reversible depression of cerebral electrical activity in the two cases reported. Until further experience with the determination of brain death in children is acquired, criteria accepted for adults should not be presumed to be valid.

The authors wish to thank Mrs. Bertha Pfisterer and Mr. Galen Schmucker (EEG technicians) for their meticulous attention to detail in the EEG recordings. Dr. Jan Muller performed the neuropathological examination in both cases.

\section{REFERENCES}

Becker, D. P., Robert, C. M. Jr., Nelson, J. R., and Stern, W. E. (1970). An evaluation of the definition of cerebral death. Neurology, 20, 459-462.

Bird, T. D., and Plum, F. (1968). Recovery from barbiturate overdose coma with a prolonged isoelectric encephalogram. Neurology, 18, 456-460.

DeMyer, W., and White, P. T. (1964). EEG in holoprosencephaly (arhinencephaly). Archives of Neurology, 11, 507-520.

Harvard Medical School (1968). A definition of irreversible coma. Report of the Ad Hoc Committee to Examine the Definition of Brain Death. Journal of the American Medical Association, 205, 337-340.

Lindgren, S., Petersén, I., and Zwetnow, N. (1968). Prediction of death in serious brain damage. Acta Chirurgica Scandinavica, 134, 405-416.

Silverman, D., Saunders, M. G., Schwab, R. S., and Masland, R. L. (1969). Cerebral death and the electroencephalogram. Journal of the American Medical Association, 209, 15051510.

Silverman, D., Masland, R. L., Saunders, M. G., and Schwab, R. S. (1970). Irreversible coma associated with electrocerebral silence. Neurology, 20, 525-533.

Windle, W. F. (1964). Neurological deficits of asphyxia at birth of rhesus monkeys. Prevention and therapy. In Neurological and Electroencephalographic Correlative Studies in Infancy, pp. 329-339. Edited by P. Kellaway, and I. Petersen. Grune and Stratton: New York. 